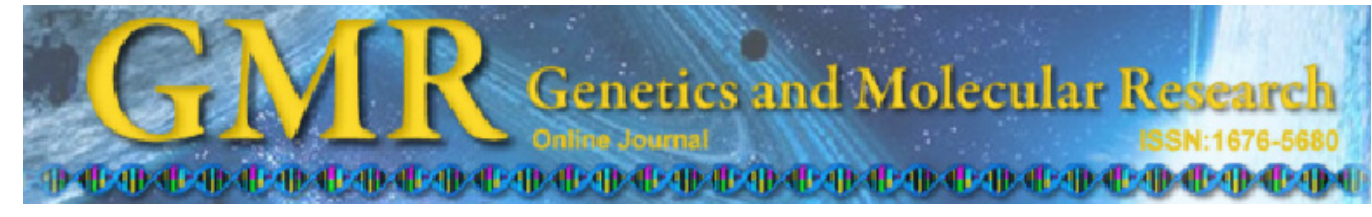

\title{
Knockdown expression of Apc11 leads to cell- cycle distribution reduction in G2/M phase
}

\author{
Y.-J. Shi and K.-K. Huo \\ State Key Laboratory of Genetic Engineering, Institute of Genetics, \\ School of Life Sciences, Fudan University, Shanghai, China \\ Corresponding author: K.-K Huo \\ E-mail:kkhuo@fudan.edu.cn / kkhuo2002@163.com
}

Genet. Mol. Res. 11 (3): 2814-2822 (2012)

Received April 19, 2012

Accepted August 7, 2012

Published August 24, 2012

DOI http://dx.doi.org/10.4238/2012.August.24.6

\begin{abstract}
Anaphase-promoting complex/cyclosome (APC/C) is a key E3 ubiquitin ligase in cell division, which catalyses ubiquitination of cell-cycle regulators. Studying this complex could reveal important information regarding its application in cancer research and therapy. In this study, 4 synthesized small interfering RNAs (siRNAs) were transfected into HEK293T cells to suppress messenger RNA (mRNA) of Apc11; 2 of these reduced the amount of Apc11 mRNA by over $50 \%$. Further experiments showed that rather than causing apoptosis, siRNA transfection led to cell-cycle distributions characterized by less time spent in G2/M phase and more time spent in G1 phase. This phenomenon was specifically induced by Apc11 silencing, as co-transfection of siRNA and an Apc11 plasmid could reverse this distribution bias. Our results suggested that siRNA targeted against Apc11 could hamper entry into G2/M phase. Current efforts are focused on elucidating the function and utility of the APC complex for clinical applications.
\end{abstract}

Key words: siRNA; Anaphase-promoting complex; Cell proliferation; Cell cycle 


\section{INTRODUCTION}

Continuous cell division, caused by deregulation, is a widely shared characteristic among cancer cells. Strategies targeting different stages of the cell cycle have been proposed by researchers throughout the world. Mitosis-targeting strategies have attracted increasingly more attention, as it is believed that the apoptotic network is linked to mitotic duration, which suggests that maximizing the time required for cells to exit mitosis could be a potential approach for cancer therapy (Huang et al., 2010). Drugs targeting the spindle assembly checkpoint (SAC) have been shown to be effective in a broad range of cancers (Wood et al., 2001; Matson and Stukenberg, 2011). The SAC ensures equal and reliable chromosome segregation during cell division (Bharadwaj and Yu, 2004; Musacchio and Salmon, 2007). In instances of insufficient or unequal kinetochore-microtubule attachments, the SAC inhibits anaphase onset; the SAC also recruits checkpoint proteins to unattached kinetochores. The anaphase-promoting complex/cyclosome (APC/C) is suppressed by binding to checkpoint proteins including Mad2 (mitotic arrest deficit 2), Bub1 $\beta$ (budding uninhibited by benzimidazoles 1 homolog beta) and Bub3 (budding uninhibited by benzimidazoles 3 homolog). When all kinetochores are properly attached to the microtubule, $\mathrm{APC} / \mathrm{C}$ is released to drive cells into anaphase by inducing the degradation of securin and cyclin B, leading cells into anaphase and exiting mitosis. By binding to microtubules, spindle poisons maintain SAC activity and arrest mitosis, which leads to cell death (Rieder and Maiato, 2004). However, resistance/escape (Brito and Rieder, 2006; Gascoigne and Taylor, 2008) to these drugs and neurotoxicity (Jordan and Wilson, 2004) are reported as challenges in clinical practices. Leaky degradation of APC/C substrates required for mitosis exit with activated SAC and non-specific toxicity demand improvement; therefore, targeting the $\mathrm{APC} / \mathrm{C}$ might be a potential alternative approach.

$\mathrm{APC} / \mathrm{C}$ is a multisubunit E3 ubiquitin ligase, whose activity oscillates during the cell cycle to result in ubiquitination of different regulatory proteins, followed by $26 \mathrm{~S}$ protease degradation. Substrates of human APC/C include cyclin B, cyclin A, securin, and aurora kinase A (Park et al., 2005). Targeting the activator Cdc20 (cell division cycle 20) with small interfering RNA (siRNA) has been demonstrated to be effective for inducing apoptosis in cancer cells (Huang et al., 2009); thus, it is important to understand whether targeting the catalytic core subunit could be an alternative therapeutic option. Apc11 (anaphase promoting complex subunit 11) is a conserved really interesting new gene (RING)-H2-finger protein, responsible for catalyzing ubiquitin-chain formation. In previous papers, associations have been inferred between dysfunctional Apc11 function and mitosis arrest. Hydrogen peroxide was able to bind Apc11, which was followed by cell-cycle arrest (Chang et al., 2004). Silencing the Apc11 gene resulted in embryonic death in nematode (Moore and Boyd, 2004). Furthermore, no mutation in Apc11 has been reported in cancer samples, as it is believed that such a mutation would result in cell death (Wang et al., 2003).

RNA interference (RNAi) is a post-transcriptional gene-silencing mechanism in which a double-stranded RNA is introduced into cells and inhibits the expression of a particular gene by degrading the homologous mRNA sequences. In this study, synthesized siRNA was transfected into HEK293T cells to knock down Apc11 mRNA expression. Information regarding the effect and impact of Apc11 gene silencing on cell proliferation and the cell cycle may contribute to further research on APC complex function and possible future applications. 


\section{MATERIAL AND METHODS}

\section{Cell culture}

HEK293T cells were purchased from the American Type Culture Collection. Cells were grown at $37^{\circ} \mathrm{C}$ in humid air containing $5 \% \mathrm{CO}_{2}$ and cultured in Dulbecco's modified Eagle's medium (Gibco, Life Technologies, Carlsbad, CA, USA) supplemented with $10 \%$ fetal calf serum, $100 \mathrm{U} / \mathrm{mL}$ penicillin, and $100 \mu \mathrm{g} / \mathrm{mL}$ streptomycin (Sigma, St. Louis, MO, USA). Cells were digested once they reached over $95 \%$ confluence with $0.05 \%$ Trypsin (Gibco). The medium was changed every 2 days.

\section{Transient transfection}

HEK293T cells were seeded onto 96-well microplates (1000 cells/well) in $100-\mu \mathrm{L}$ antibiotic-free culture medium. Twenty-four hours after seeding, cells were transfected with siRNA according to manufacturer instructions. Plasmid DNA $(0.2 \mu \mathrm{g})$ and $0.5 \mu \mathrm{L}$ Lipofectamine 2000 (Invitrogen, Carlsbad, CA, USA) were first pre-mixed in 50- $\mu \mathrm{L}$ antibioticfree OPTI-MEM (Invitrogen) to generate transfection complexes (60 min at room temperature), and were then added to the cells. After $24 \mathrm{~h}$, the complexes were removed by fresh culture medium supplemented with antibiotics.

\section{siRNA interference}

The siRNA was designed and synthesized by the GeneChem Company (Montreal, Quebec, Canada). Four target sequences were designed at the coding region ( $\mathrm{C} 1$ and $\mathrm{C} 2$ ) and the untranslated region (U1 and U2), according to nucleotide information obtained from the National Center for Biotechnology Information (NCBI). These sequences are shown in Table 1. Fifty pM siRNA was used for each transfection on a 24-well plate.

\section{Reverse transcription-polymerase chain reaction (RT-PCR) assay}

Total RNA was extracted from cells with Trizol (Invitrogen). The Oligo $\mathrm{dT}_{18}$ primer was synthesized by Invitrogen. After quantification, $2 \mu \mathrm{g}$ total RNA was used to perform RT-PCR according to the Moloney Murine Leukemia Virus (MMLV)-RT manual (Promega, Madison, WI, USA). PCR was performed using $100 \mathrm{ng}$ cDNA and Taq DNA polymerase (Takara, Shiga, Japan). The PCR program consisted of: $95^{\circ} \mathrm{C}$ at $10 \mathrm{~min}$ followed by 25 cycles of $95^{\circ} \mathrm{C}$ for $40 \mathrm{~s}, 60^{\circ} \mathrm{C}$ for $15 \mathrm{~s}$ and $72^{\circ} \mathrm{C}$ for $60 \mathrm{~s}$, and a final stage at $72^{\circ} \mathrm{C}$ for $5 \mathrm{~min}$. The PCR product was run on a $\%$ agarose gel stained with ethidium bromide. Apc11 was amplified with Apc11F (5'-ATGAAGGTGAAGATTAAGT-3') and Apc11R (5'-TCACTCCTTGA ACTTCCATT-3'). GAPDH was amplified with GAPDH F (5'-TGAAGGTCGGAGTCAAC GGATTTGGT-3') and GAPDH R (5'-CATGTGGGCCATGAGGTCCACCAC-3'). All primers were designed with the Primer 5 software. 


\section{Gene cloning}

Human Apc11 was cloned from the CloneTech (Mountain View, CA, USA) yeast 2-hybrid human fetal liver library. The primer sequences were: forward primer, 5'-ATGAAGGTGA AGATTAAGT-3'; reverse primer, 5'-TCACTCCTTGAACTTCCATT-3'. The amplified fragment was cloned into a T vector (Takara). After transformation into DH5 $\alpha$ competent cells, the extracted plasmid sequence was confirmed by sequencing. Then, Apc11 was cloned into a pCMV-HA vector after digestion with EcoRI and NotI. The confirmed Apc11 sequence was amplified again with the forward primer, 5'-CCGAATTCGATGAAGGTGAAGATTAAGT-3' and reverse primer, 5'-TTGCGGCCGCTCACTCCTTGAACTTCCATT-3', followed by EcoRI and NotI digestion and ligation into a pCMV-HA vector.

\section{Fluorescence-activated cell sorting (FACS) assay}

The siRNA was transfected along with pCMV-HA or pCMV-HA-Apc11 to assess its effect on cell-cycle distribution and specificity. Cells were collected $60 \mathrm{~h}$ after transient transfection. Briefly, after 2 washes with phosphate-buffered saline (PBS), cells were treated with $0.25 \%$ Trypsin. The collected cells were centrifuged at $1500 \mathrm{~g}$ for 10 min to remove the supernatant. The cells were fixed in $1 \mathrm{~mL} 75 \%$ ethanol at $4{ }^{\circ} \mathrm{C}$ for at least $2 \mathrm{~h}$. After removing the ethanol, $500 \mu \mathrm{L}$ propidium iodide (PI) dye was added for nucleic acid staining. After at least $15 \mathrm{~min}$, cells were filtered through mesh nylon membrane and cell-cycle distribution of the whole-cell population was analyzed by FACS on BD Avian Cytometry.

\section{RESULTS}

In our study, siRNA against Apc11 was transfected into HEK293 cells. Seven different transcript isoforms were identified as Apc11 mRNAs. Six of these encode the same 85-amino acid RING finger protein-APC11, and the last encodes a 197-amino acid protein of unknown function. Alignment showed that all mRNA shared the same sequence in two region, encoding 85-amino acid RING finger protein-APC11 and the 3'-untranslated region (3'-UTR) and 2 siRNA were designed in each region.

In addition to the 4 different siRNAs against Apc11, siRNA against GAPDH mRNA was used as a positive control, and random non-specific siRNA was used as a negative control. According to the NCBI website, the human Apc11 gene has 7 different isoforms of transcripts that can be translated into 2 different proteins, as shown in Figure 1. Among these proteins, only the short protein has an intact RING finger domain, which is a highly conserved structure in eukaryotes. The other 6 isoforms encode the protein for the APC/C catalytic core. All transcript isoforms share common sequences in the 3'-untranslated region and the protein coding region. Specific sequences are shown in Table 1; $\mathrm{C} 1$ and $\mathrm{C} 2$ were designed according to coding sequence and $\mathrm{U} 1$ and $\mathrm{U} 2$ were designed according to the untranslated region sequence. NM_001002244.1 does not have an intact RING finger structure, although it shares the target sequence of the other 6 isoforms. 

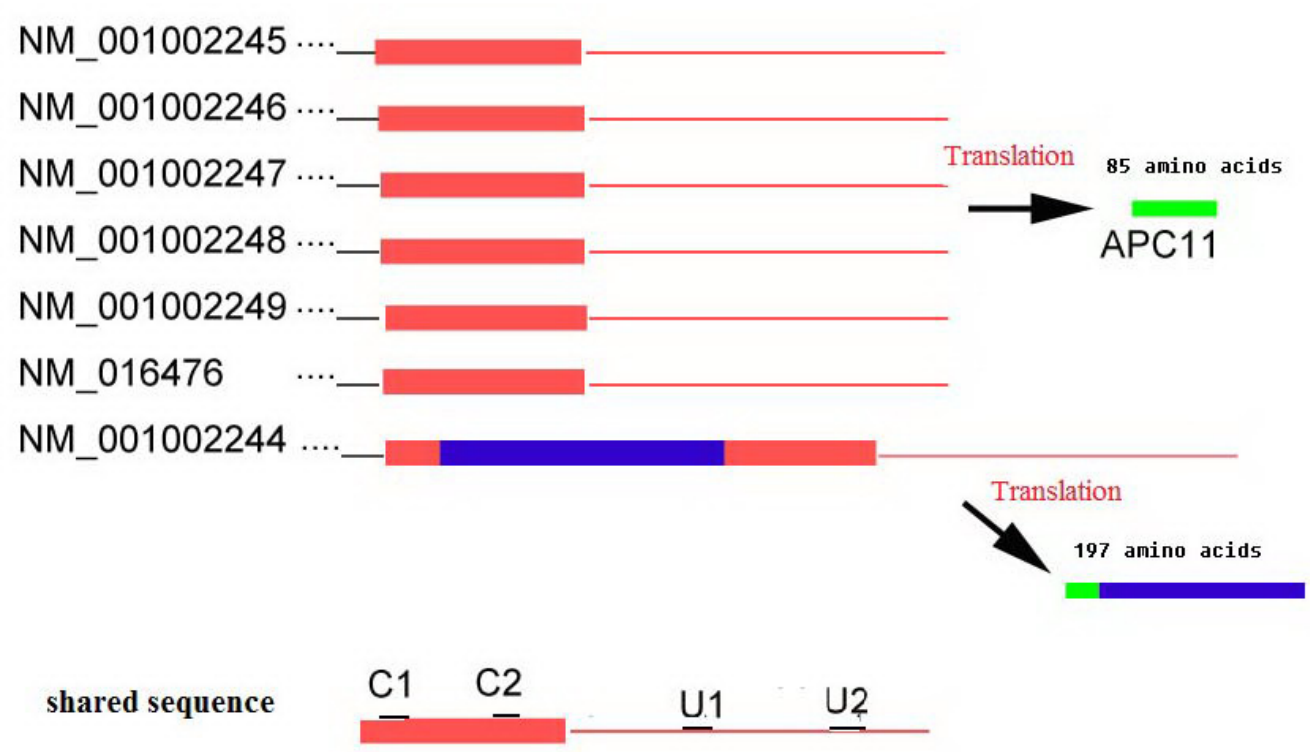

Figure 1. Alignment of human anaphase promoting complex subunit 11 (Apc11) mRNA. Seven different transcript isoforms were identified as Apc11 mRNA. Six of them encode the same RING-H2-finger protein-Apc11 of 85 amino acids in length and the last one encodes a protein of 197 amino acids unknown for any function. Alignment is performed with the Clustal software. All mRNA share the same sequence encoding 85 amino acids and 3'-untranslated region, shown in figure as pink blocks and pink lines. The position of target sequence is shown on shared sequence not in precise proportion of actual position (black dots and lines stand for 5'-untranslated region; pink and blue blocks stand for coding sequence; pink lines stand for 3'-untranslated region; translation means protein synthesis from mRNA; green and blue slim blocks represent proteins).

\begin{tabular}{ll} 
Table 1. siRNA sequences. \\
\hline U1/UTR-130 \\
U2/UTR-265 \\
C1/CDS-45 & 5'-CCAUCACUAUGUUGACACUTTAGUGUCAACAUAGUGAUGGTT-3' \\
C2/CDS-228 & 5'-GGGUGGCCAACGAUGAGAATTUUCUCAUCGUUGGCCACCCAG-3' \\
-/random sequence & 5'-GCCAGGAAUGGAAGUUCAATTUUGAACUUCCAUUCCUGGCGG-3' \\
+/GAPDH & 5'-UUCUCCGAACGUGUCACGUTTACGUGACACGUUCGGAGAATT-3' \\
\hline
\end{tabular}

Forty-eight hours after transfection, total RNA was extracted from cells. After RT-PCR and PCR amplification, the final product is shown in Figure 2. Apc11 mRNA was reduced by over $50 \%$ in the $\mathrm{U} 2$ and $\mathrm{C} 1$ group, with $G A P D H$ as a loading control. The observed decrease in the GAPDH-positive control confirmed that the reaction system was working. The negative control showed the baseline quantity of Apc11 without specific interference. From the electrophoresis results, we confirmed that siRNA could inhibit Apc11 mRNA expression in HEK293 cells, with $\mathrm{C} 1$ reaching 80\% inhibition and U2 reaching $50 \%$ inhibition. The other transfectants, $\mathrm{U} 1$ and $\mathrm{C} 2$, showed minor suppression, decreasing only $10-20 \%$. 


\section{C1 1 C2 1 U1 2 2 -+

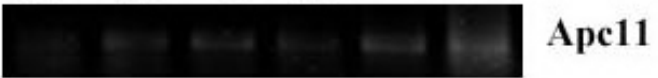

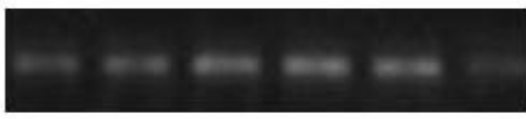

\section{GAPDH}

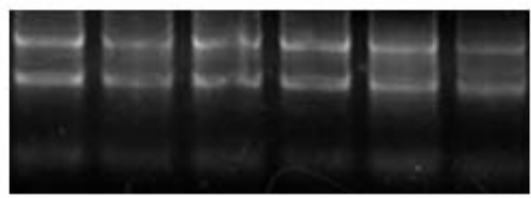

\section{Total RNA}

Figure 2. Anaphase promoting complex subunit 11 (Apc11) expression level changes after siRNA transfection. Greater than $50 \%$ reduction was observed in the $\mathrm{U} 2$ and $\mathrm{C} 1$ group. GAPDH was used as a loading control. All total RNA was confirmed to be of similar quality prior to RT-PCR.

To investigate the mechanism of siRNA inhibition in the present study, transfected cells were stained with PI dye to assess the cell-cycle distribution. Surprisingly, apoptosis was not detected. As shown in Figure 3, $60 \mathrm{~h}$ after transfection, the cell-cycle distribution showed less percentage in $\mathrm{G} 2 / \mathrm{M}$ phase compared to the negative control group. This phenomenon was specifically caused by $\mathrm{U} 2$ or $\mathrm{C} 1$; when the plasmid with coding sequence of Apc11 was co-transfected with siRNA, increases in the G2/M phase and decreases in the G1 phase were observed. The comparison to the random siRNA group clearly implied that overexpression of Apc11 led to increased cell-cycle distribution in G2/M phase.

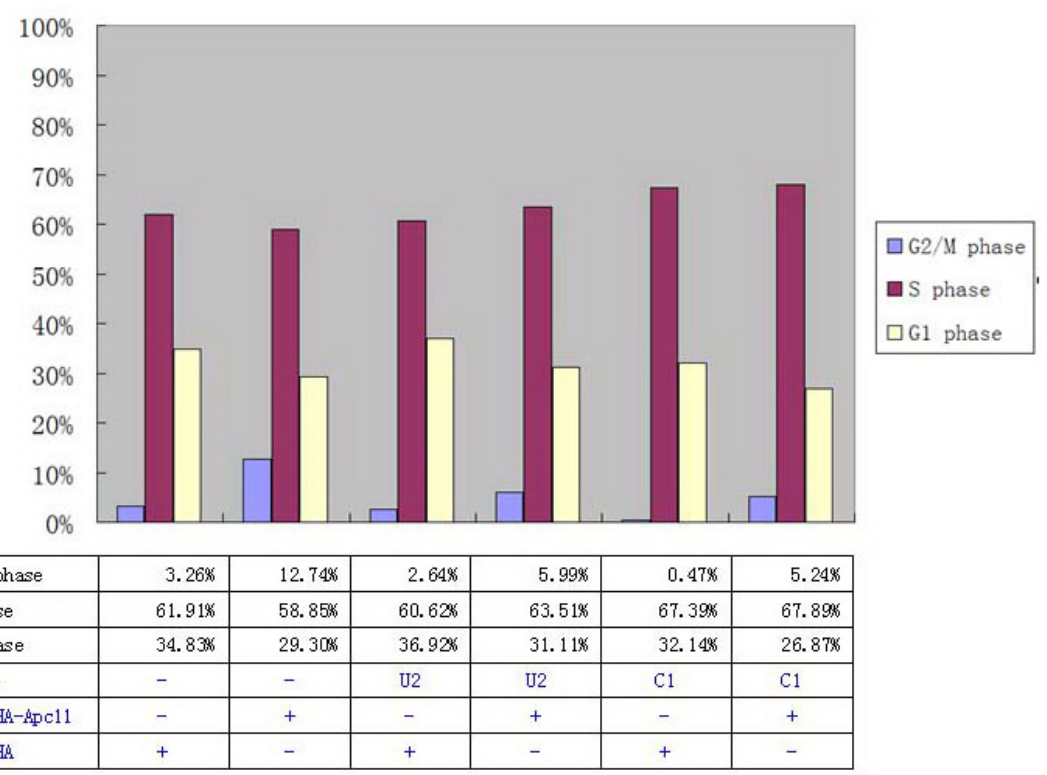

Figure 3. The G2/M phase distribution decreased after transfection of siRNA against anaphase promoting complex subunit 11 (Apc11). Exogenous Apc11 could rescue this phenomenon. Comparison between vector and exogenous Apc11 in the same siRNA treatment showed similar trend toward the increase in G2/M phase and decrease in G1 phase. 


\section{DISCUSSION}

From the current view, APC is a 4-part enzyme complex, including a structural complex (anaphase promoting complex subunit 1/anaphase promoting complex subunit 4/anaphase promoting complex subunit 5), a tetratricopeptide repeat (TPR) arm that functions primarily for activator binding (anaphase promoting complex subunit 8/anaphase promoting complex subunit 6/anaphase promoting complex subunit 3/anaphase promoting complex subunit 7), the activators that recruit substrates (cell division cycle-associated protein 20/cadherin1, in short Cdc20/Cdh1), and a catalytic arm that houses the E2-binding sites (anaphase promoting complex subunit 2/Apc11/anaphase promoting complex subunit 10) (Thornton and Toczyski, 2006). Previous reports describe the role of APC/C in cancer as a paradox. Mutations in the structural complex and TPR arm are reported in cancer samples and cancer cell lines and are implied as the cause for genomic instability (Wang et al., 2003; Park et al., 2005). To circumvent resistance to microtubule poison, also known as slippage, which is actually leaky degradation of cyclin B with activated SAC, Huang et al. (2009) reported using siRNA against $\mathrm{Cdc} 20$ as a promising therapeutic approach for cancer. This approach resulted in delayed cyclin B degradation and intrinsic apoptosis in cancer cell lines, including the HeLa cell line (human cervix epithelial adenocarcinoma), MCF cell line (human metastatic breast/mammary gland epithelial adenocarcinoma) and A459 cell line (human lung cancer), which led the authors to suggest mitosis exit as a better target than SAC. A recent study showed that precocious activation of APC/C-Cdh1 during metaphase (pre-anaphase) accounted for slippage (Toda et al., 2012), which indicated that Cdh1 is a potential target for cancer therapy. At the same time, inactivated Cdh1 was required for activation of the DNA damage checkpoint (Bassermann et al., 2008; Jennings et al., 2009; Wiebusch and Hagemeier, 2010). Human Apc11 was cloned over 10 years ago, and its role as a catalytic core subunit has been demonstrated both in vitro and in vivo (Gmachl et al., 2000; Tang et al., 2001). However, no investigation to date has provided clear answers regarding whether this gene could be a target for cancer therapy.

In the present study, 4 siRNA were designed to assess the potential of Apc11, the RING-FINGER subunit of APC/C, as a target for cancer therapy. Unexpectedly, the silencing of Apc11 mRNA did not result in mitosis arrest or apoptosis. The siRNA against Apc11 led to less cell distribution in G2/M phase and more distribution in G1 phase. To confirm that this phenomenon was specifically caused by knockdown of Apc11, siRNA was transfected along with a vector containing Apc11, and reversed distribution was observed. Furthermore, overexpression of Apc11 by transfecting the vector containing Apc11 led to an accumulation of G2/M cells compared to cells transfected with an empty vector, which has not been demonstrated in previous publications.

It is unclear as to why silencing of Apc11 did not lead to mitosis arrest. One possible reason is that the mitotic substrates are more sensitive to APC/C activity, and the Apc 11 mRNA level was still above the threshold for APC/C complex. Secondly, accumulation of APC/Cdh1 substrates in G1, including Tome-1 (also known as Cdca3, cell division cycle associated 3), Skp2 (S-phase kinase-associated protein 2), and Ets2 (also known as Tert, telomerase reverse transcriptase) could be another reason for this result. By associating with SCF complex (Skp, Cullin, F-box containing complex), Tome-1 regulates G2/M transition through proteolysis of Wee1. Accumulation of Tome-1 in G1 phase leads to degradation of Wee1, halting cell-cycle progression (Ayad et al., 2003). As an F-box protein, Skp2 mediates recognition of p21 and 
p27, followed by ubiquitination by the SCF complex (Tsvetkov et al., 1999). In addition, degradation of Ets2 has been reported to limit cyclin D expression (Albanese et al., 1995). Without functional APC/Cdh1, Skp2 accumulation leads to degradation of p21 (also known as cyclin-dependent kinase inhibitor 1A) and p27 (also known as Sjogren syndrome/scleroderma autoantigen 1); Ets2 accumulation leads to cyclin D1 elevation, which hampers transition into $\mathrm{S}$ phase. Thirdly, dysfunctional APC/Cdh1 might trigger the DNA damage checkpoint. Premature entry into $\mathrm{S}$ phase as a consequence of failure to inactivate the cyclin-cdk complex in G1 phase results in unscheduled origin firing, which leads to slow replication initiating from fewer origins. This phenomenon leads to increased stalled replication forks and the possibility of replication errors, which then might activate the p53 (tumor protein p53)/p21-dependent replication checkpoint for G1 phase delay (Vaziri et al., 2003).

A recent report from Chow's group found that $U$ cells that were co-depleted of both $\mathrm{Cdc} 20$ and $\mathrm{Cdh} 1$ could complete mitosis, and they suggested that the inhibition of cyclin B1CDK1 (cyclin-dependent kinase 1) might be an alternative mechanism to APC-derived ubiquitination (Chow et al., 2011). Further efforts are being made with the goal of understanding the mechanism for G2/M reduction after silencing Apc11. The results of these studies would provide more information regarding cell-cycle mechanisms that would allow for further investigation of this potential cancer therapy strategy.

\section{ACKNOWLEDGMENTS}

Research supported by the China High Technology Research Program Grant (\#2006AA02A310, \#2004BA711A19).

\section{REFERENCES}

Albanese C, Johnson J, Watanabe G, Eklund N, et al. (1995). Transforming p21 ras mutants and c-Ets-2 activate the cyclin D1 promoter through distinguishable regions. J. Biol. Chem. 270: 23589-23597.

Ayad NG, Rankin S, Murakami M, Jebanathirajah J, et al. (2003). Tome-1, a trigger of mitotic entry, is degraded during G1 via the APC. Cell 113: 101-113.

Bassermann F, Frescas D, Guardavaccaro D, Busino L, et al. (2008). The Cdc14B-Cdh1-Plk1 axis controls the G2 DNAdamage-response checkpoint. Cell 134: 256-267.

Bharadwaj R and Yu H (2004). The spindle checkpoint, aneuploidy, and cancer. Oncogene 23: 2016-2027.

Brito DA and Rieder CL (2006). Mitotic checkpoint slippage in humans occurs via cyclin B destruction in the presence of an active checkpoint. Curr. Biol. 16: 1194-1200.

Chang TS, Jeong W, Lee DY, Cho CS, et al. (2004). The RING-H2-finger protein APC11 as a target of hydrogen peroxide. Free Radic. Biol. Med. 37: 521-530.

Chow JP, Poon RY and Ma HT (2011). Inhibitory phosphorylation of cyclin-dependent kinase 1 as a compensatory mechanism for mitosis exit. Mol. Cell. Biol. 31: 1478-1491.

Gascoigne KE and Taylor SS (2008). Cancer cells display profound intra- and interline variation following prolonged exposure to antimitotic drugs. Cancer Cell 14: 111-122.

Gmachl M, Gieffers C, Podtelejnikov AV, Mann M, et al. (2000). The RING-H2 finger protein APC11 and the E2 enzyme UBC4 are sufficient to ubiquitinate substrates of the anaphase-promoting complex. Proc. Natl. Acad. Sci. U. S. A. 97: 8973-8978.

Huang HC, Shi J, Orth JD and Mitchison TJ (2009). Evidence that mitotic exit is a better cancer therapeutic target than spindle assembly. Cancer Cell 16: 347-358.

Huang HC, Shi J, Orth JD and Mitchison TJ (2010). Cell death when the SAC is out of commission. Cell Cycle 9: 20492050.

Jennings P, Aydin S, Bennett J, McBride R, et al. (2009). Inter-laboratory comparison of human renal proximal tubule 
(HK-2) transcriptome alterations due to Cyclosporine A exposure and medium exhaustion. Toxicol. In Vitro 23: 486-499.

Jordan MA and Wilson L (2004). Microtubules as a target for anticancer drugs. Nat. Rev. Cancer 4: 253-265.

Matson DR and Stukenberg PT (2011). Spindle poisons and cell fate: a tale of two pathways. Mol. Interv. 11: 141-150.

Moore R and Boyd L (2004). Analysis of RING finger genes required for embryogenesis in C. elegans. Genesis 38: 1-12. Musacchio A and Salmon ED (2007). The spindle-assembly checkpoint in space and time. Nat. Rev. Mol. Cell Biol. 8: 379-393.

Park KH, Choi SE, Eom M and Kang Y (2005). Downregulation of the anaphase-promoting complex (APC)7 in invasive ductal carcinomas of the breast and its clinicopathologic relationships. Breast Cancer Res. 7: R238-R247.

Rieder CL and Maiato H (2004). Stuck in division or passing through: what happens when cells cannot satisfy the spindle assembly checkpoint. Dev. Cell 7: 637-651.

Tang Z, Li B, Bharadwaj R, Zhu H, et al. (2001). APC2 Cullin protein and APC11 RING protein comprise the minimal ubiquitin ligase module of the anaphase-promoting complex. Mol. Biol. Cell 12: 3839-3851.

Thornton BR and Toczyski DP (2006). Precise destruction: an emerging picture of the APC. Genes Dev. 20: 3069-3078.

Toda K, Naito K, Mase S, Ueno M, et al. (2012). APC/C-Cdh1-dependent anaphase and telophase progression during mitotic slippage. Cell Div. 7: 4.

Tsvetkov LM, Yeh KH, Lee SJ, Sun H, et al. (1999). p27(Kip1) ubiquitination and degradation is regulated by the SCF(Skp2) complex through phosphorylated Thr187 in p27. Curr. Biol. 9: 661-664.

Vaziri C, Saxena S, Jeon Y, Lee C, et al. (2003). A p53-dependent checkpoint pathway prevents rereplication. Mol. Cell 11: 997-1008.

Wang Q, Moyret-Lalle C, Couzon F, Surbiguet-Clippe C, et al. (2003). Alterations of anaphase-promoting complex genes in human colon cancer cells. Oncogene 22: 1486-1490.

Wiebusch L and Hagemeier C (2010). p53- and p21-dependent premature APC/C-Cdh1 activation in G2 is part of the long-term response to genotoxic stress. Oncogene 29: 3477-3489.

Wood KW, Cornwell WD and Jackson JR (2001). Past and future of the mitotic spindle as an oncology target. Curr. Opin. Pharmacol. 1: 370-377. 